Article

\title{
Characterization of Potential Micrometeorites by Synchrotron Analysis
}

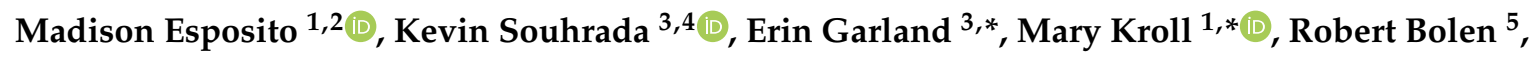 \\ Victoria Hernandez ${ }^{6}$, Janet Kaczmarek ${ }^{7}$, David Meisel ${ }^{8}$, Anya Swiss ${ }^{6}$, Paul Northrup ${ }^{9, *(D)}$, \\ Vivian Stojanoff ${ }^{10}$, Juergen Thieme ${ }^{10}$ and Aleida Perez ${ }^{10, *(\mathbb{D})}$ \\ 1 Science Department, West Islip High School, West Islip, NY 11795, USA; espomaddie@gmail.com \\ 2 Department of Chemistry, Princeton University, Princeton, NJ 08544, USA \\ 3 Science Department, Bay Shore High School, Bay Shore, NY 11706, USA; kcsouhrada@gmail.com \\ 4 Department of Physics, Cornell University, Ithaca, NY 14853, USA \\ 5 Science Department, Eastport South Manor High School, Manorville, NY 11949, USA; bolenr@esmonline.org \\ 6 Science Department, William Floyd High School, Mastic Beach, NY 11951, USA; \\ victoria.dambrosia@gmail.com (V.H.); a.lisa.swiss@gmail.com (A.S.) \\ 7 Science Department, Sayville High School, Sayville, NY 11782, USA; kaczmarekjanet@gmail.com \\ 8 Department of Physics and Astronomy, Geneseo University, Geneseo, NY 14454, USA; \\ proximab@geneseo.edu \\ 9 Department of Geosciences, Stony Brook University, Stony Brook, NY 11794, USA \\ 10 Brookhaven National Laboratory, Upton, NY 11973, USA; stojanof@bnl.gov (V.S.); jthieme@bnl.gov (J.T.) \\ * Correspondence: egarland@bayshore.k12.ny.us (E.G.); m.kroll@wi.k12.ny.us (M.K.); \\ paul.northrup@stonybrook.edu (P.N.); pereza@bnl.gov (A.P.)
}

Received: 29 May 2020; Accepted: 14 July 2020; Published: 16 July 2020

check for updates

\begin{abstract}
Micrometeorites (MMs) are small particles that account for most of the extraterrestrial material deposited on Earth. Synchrotron X-ray fluorescence and diffraction allowed for chemical and mineral characterization to distinguish MM from atmospheric particulate. The relative components of iron, nickel, and other elements were considered in the identification of ferrous MM while high amounts of titanium were considered an indication that the particles were of atmospheric origin. Out of 100 samples collected by high school students and teachers, eight were taken to a synchrotron for analysis. Of those eight, three exhibited extraterrestrial compositions. X-ray absorption near-edge structure analysis revealed that the same three samples contained sulfide, the main sulfur form constituent in MM. X-ray microdiffraction analysis showed the presence of the minerals pentlandite and forsterite. Collectively, these results support the extraterrestrial nature of the three particles.
\end{abstract}

Keywords: micrometeorites; meteorites; synchrotron; X-ray fluorescence; X-ray absorption spectroscopy; X-ray diffraction; $\mathrm{X}$-ray absorption near edge structure

\section{Introduction}

Micrometeorites (MMs) are a subset of cosmic dust particles that compose most of the extraterrestrial material reaching Earth, making MMs integral in researching extraterrestrial chemistry and Earth's geochemical supply of certain elements. These particles are significant factors in extraterrestrial chemistry in our solar system due to their abundance and omnipresence.

MM mineralogy and textures are a combination of parent body features mixed with phases formed by flash heating and quench cooling [1]. Micrometeorites can reach up to $3 \mathrm{~mm}$ in diameter [2] but are most commonly $250 \mu \mathrm{m}$ [3]. Estimates of MM mass flux are subtle and varied. Suttle and Folco (2020) provided a recent summary of estimates based on some of the most prominent collections [3]. Their estimate lies at $1555 \pm 753 \mathrm{t}$ /year, based on samples from the collection trap TAM65 of the 2017-2018 
Italian Antarctic Campaign of the Programma Nazionale delle Ricerche [3]. Most modern MM flux estimates fall within an order of magnitude of this [4-9]. These estimates have significant margins of error due to many factors, including weathering effects, inefficient collection methods, short accumulation windows, or small sample sizes. Genge et al. (2008) established the most current classification system for MMs [1]. Firstly, MMs are identified as melted, partially melted, or unmelted [1]. Relevant to this study are the melted MMs, classified as cosmic spherules (CSs) due to the spherical shape they obtain from atmospheric entry [1]. Cosmic spherules can further be categorized as iron-rich (I-type), glassy with magnetite (G-type), or silicate (S-type), with S-type being by far the most abundant [1]. Identifying petrographic features of CSs include significant fusion of pre-atmospheric entry phases and the presence of vesicles [1]. There is also a broad range of common or identifying features among the CS subtypes, such as chondritic composition for S-types or a single large central void in I-types [1]. Taylor et al. (2012) suggests that the majority of MMs come from fine-grained precursors with compositions similar to $\mathrm{CI}$ and CM meteorite classes [7]. Silicates and sulfides present in CSs can be traced to remains from early planet formation [10]. Micrometeorites of all classes can contain relict grains that have survived atmospheric entry unmelted [11]. Examining the trace elements of relict olivines and pyroxenes can illuminate connections to extraterrestrial origin sources [12,13]. Relict grains are most commonly Mg-rich olivine (forsterite), followed by Fe-rich olivine and Mg-rich low-Ca pyroxenes [11]. Pentlandite presence is associated with the sulfidization of iron and nickel [14].

Genge et al. (2017) analyzed 500 urban MMs collected by amateur meteor collector Jon Larsen's Project Stardust from roof gutters in Oslo, Norway [15]. Of the $500 \mathrm{MMs}$ collected, 48 were isolated for SEM and electron microprobe analysis and determined to be S-type cosmic spherules with identical chemistry, mineralogy, and texture to cosmic spherules collected in remote locations across Earth, such as Antarctica [15]. These findings verified the hypothesis that MMs can be collected in urban environments. Larsen's Project Stardust further continued, and it has provided an accessible introduction to micrometeorites for non-scientists [16]. Larsen collected and identified anthropogenic contaminants (such as welding spherules and glass spherules from urban road dust) posing as MM and cosmic spherules, analyzing their chondritic chemistry and textures to distinguish extraterrestrial from terrestrial [16]. Additionally, in the United States, citizen scientist, Scott Peterson, has collected urban MMs, verifying his findings through SEM analysis at the University of Minnesota [17]. Similarly, scientists from the Museum für Naturkunde Berlin and the Freie Universität Berlin engaged the citizens of Berlin in a citizen science project, collecting MMs city-wide from rooftops [18]. Kilograms of dust were sifted to visually isolate particles of interest before authentication of extraterrestrial origin with an electron microscope; over $60 \mathrm{MMs}$ have been confirmed, furthering the validity of urban MM collection and providing a model for citizen science projects involving MMs [18]. As a result, a misconception has formed in popular culture concerning the ease with which MMs can be collected in populated environments.

Comparable to what was explored by Genge et al. (2017) and Blake et al. (2018), this research utilized simple collection methods to gather particles in accessible locations rather than expeditions to polar regions or the deep sea $[15,19]$. Some recent studies have used synchrotron technology to investigate the properties of micrometeorites. Taylor et al. (2011) found that synchrotron computed microtomography was an effective non-destructive means to investigate sulfur in micrometeorites [20]. Van Maldeghem et al. (2018) used synchrotron techniques X-ray fluorescence (XRF) and X-ray diffraction (XRD) to characterize micrometeorites collected in the transantarctic and on mountain summits [21]. Dionnet et al. (2020) used synchrotron X-ray computed tomography (X-CT) to analyze giant MMs, allowing for non-destructive characterization based on the texture and visualization of porosity, which infers atmospheric entry [22]. This study supports the viability of synchrotron techniques as an alternative to SEM analysis [22]. This study uniquely examined suburban micrometeorites utilizing synchrotron technology and combined both research and educational objectives. We collected atmospheric particles locally in a simple manner and determined their origin by non-destructive synchrotron techniques. Unlike previous studies, we examined the origin of these locally collected 
atmospheric particles by non-destructive synchrotron techniques XRF, XRD, and X-ray absorption near-edge structure (XANES). In addition to gaining knowledge about MM collection, classification, chemistry, and their role in our solar system, students involved also gain hands-on experience collaborating in a team setting. Blake et al. (2018) cite this method of learning as project-based learning (PBL); learning occurs outside of the traditional classroom experience, instead focusing on collaborative approaches to conduct investigations and answer questions. PBL is accepted as a successful method to improve student learning [19].

\section{Materials and Methods}

\subsection{Micrometeorite Collection and Selection}

Micrometeorite samples were collected using two-gallon buckets on five school rooftops located on Long Island in New York, USA: West Islip High School (WI) [40.701376, -73.302859], Bay Shore High School (BS) [40.732386, -73.253736], Sayville High School (S) [40.734957, -73.094006], Longwood High School (LW) [40.864007, -72.926250], and Eastport-South Manor High School (ESM) [40.830587, -72.823288] (Figure 1). Approximately 10 buckets were left on school rooftops for an accumulation window of two weeks to collect precipitation, dust, and micrometeorite candidate samples. This process was repeated three times to maximize the number of samples. Collection occurred during the winter months into early spring to minimize pollen, spore, and other natural terrestrial particulates.

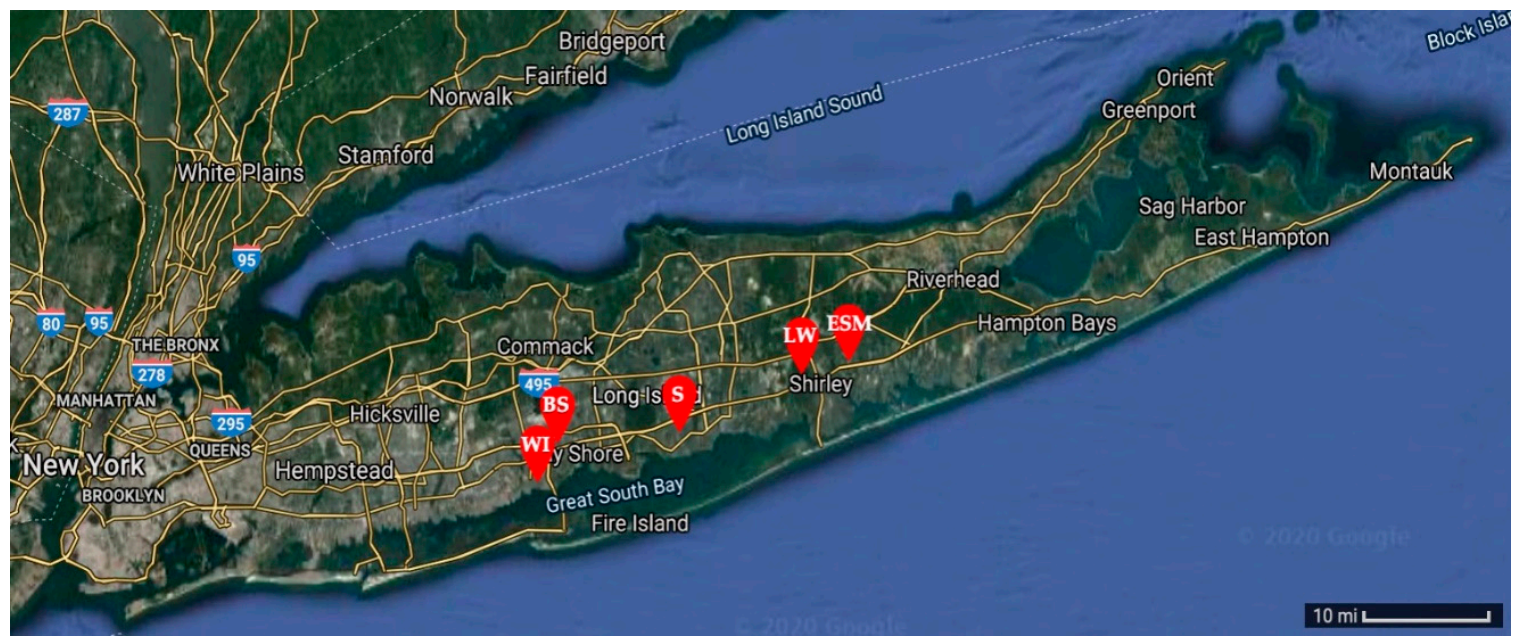

Figure 1. Potential micrometeorite samples were collected on school rooftops at West Islip High School, Bay Shore High School, Sayville High School, Longwood High School, and Eastport-South Manor High School across Long Island in New York, USA [23].

Collected precipitation was filtered through lab-grade filter paper and dried overnight at $35^{\circ} \mathrm{C}$. A neodymium magnet was then taped to the flat side of a depression glass slide. The slide was slowly moved over the dried filter paper to collect magnetic particles (Figure 2). Collected particles were isolated and observed under a visible light microscope to find particles with a diameter of approximately 100 micrometers, a relatively spherical shape, and a glossy surface consistent with the size, shape, and texture of cosmic spherules as identified in the literature [1,24]. This selected cosmic spherules that had iron or magnetite components. A Swift M6000 digital microscope was calibrated for accurate measurements and used with Motic Images +3.0 software to measure the diameter of collected samples in the high school laboratory to isolate particles for synchrotron analysis. Additional images of mounted samples were collected at the beamline (Figure 3). 


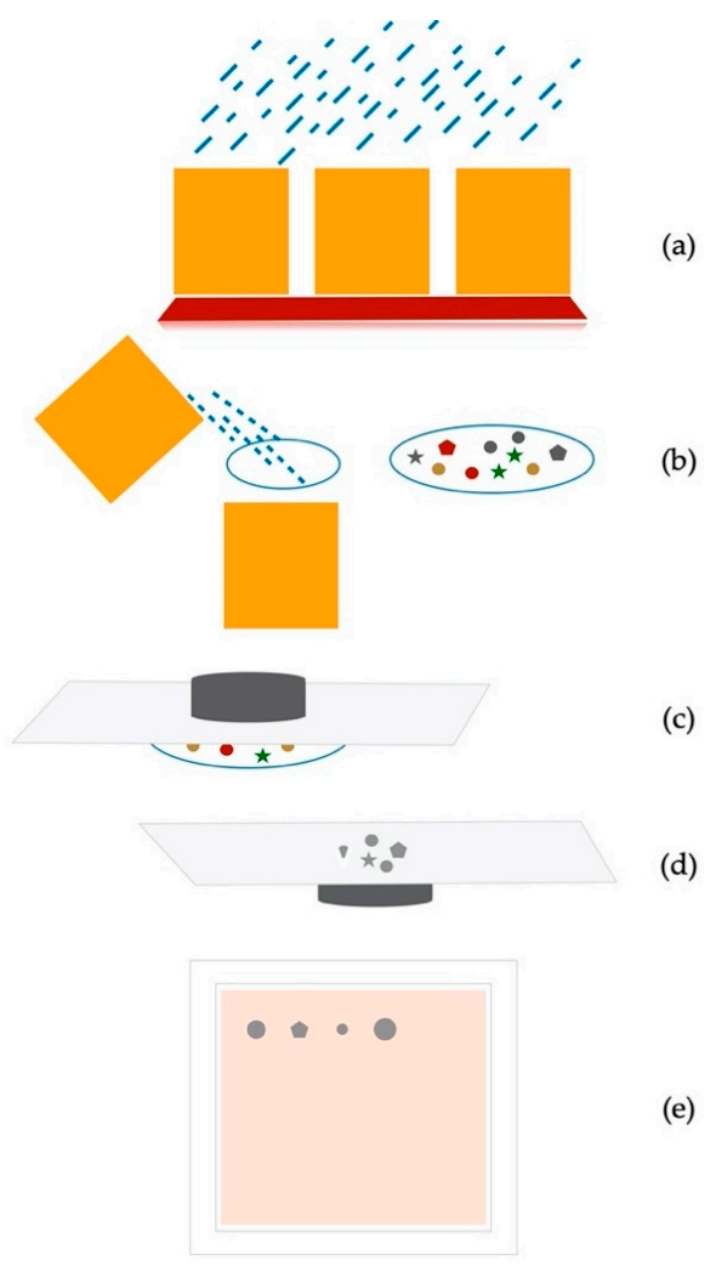

Figure 2. Atmospheric particle collection. Precipitation was collected in buckets placed on the roof tops (a); and filtered through laboratory-grade filter paper (b); particles were collected by slowly moving a magnet taped to a glass slide over the dried filter paper (c); collected particles were isolated, observed under a visible light microscope, and selected according to the diameter, shape, and texture consistent to micrometeorites (d) [1,24]; selected particles were mounted between 5- $\mu$ m-thick polypropylene sheet stretched on a cardboard frame and held in place by a thin layer of petrolatum (e). The cardboard holder allowed them to be analyzed at different beam lines.

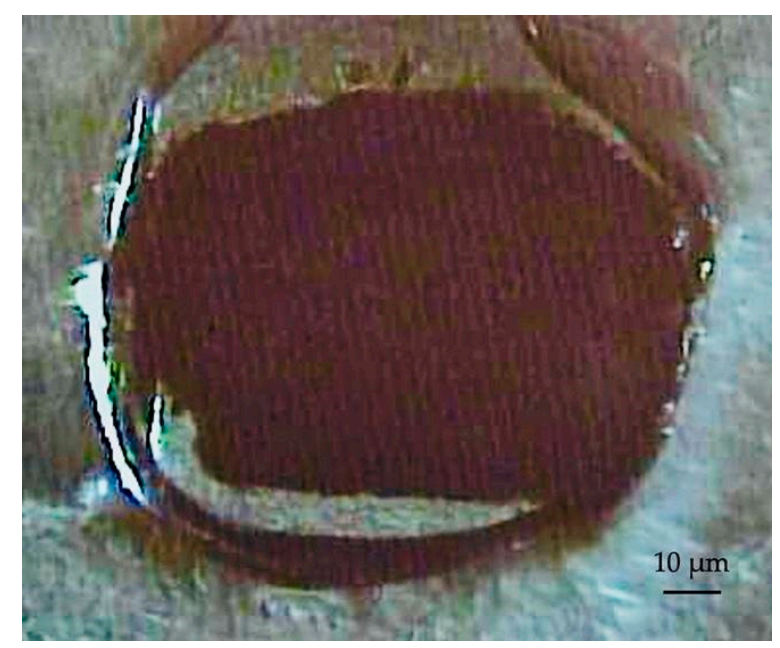

Figure 3. Optical microscopy image of the micrometeorite WestIslipB embedded in petrolatum at 100×. 


\subsection{Micrometeorite Preparation}

Meteorite pieces (e.g., Mt. Egerton (aubrite), NWA 3118 (Carbonaceous Chondrite-CC), and additional as classified in Table 1) were large enough to be mounted onto Kapton tape covering a 3-D printed plastic mold so that the meteorite surface was exposed to the $\mathrm{X}$-rays with no interference from the mounting materials. The mounting was appropriate for analysis at all respective beam lines. Micrometeorite samples were too small for this mounting procedure; these samples were sandwiched between two layers of 5 - $\mu$ m-thick polypropylene that were coated with a small amount of petrolatum to keep the samples in place. The sandwich was then taped between two cardboard frames to be mounted at the beamline.

Table 1. Metals detected using SRX and sulfur speciation determined with XANES using TES.

\begin{tabular}{cccccccccccc}
\hline Sample ID & Location & Class & Ca & Ti & Cr & Mn & Fe & Ni & Cu & Zn & Sulfur Speciation \\
\hline Hamada & U & U & M & M & H & N & H & M & N & M & sulfide \& sulfate \\
Inkerston & U & U & M & M & M & H & H & N & N & L & unknown \\
Mt. Egerton & WA & A & M & M & M & M & H & M & M & L & unknown \\
Norton County 1 & K & A & M & N & H & H & H & M & M & M & unknown \\
Norton County 2 & K & A & M & M & M & M & H & L & L & L & unknown \\
NWA 3118 & Mo & CC & M & N & M & M & H & M & N & M & sulfide \\
NWA Morocco & U & U & M & M & N & M & H & M & M & M & unknown \\
BigRock1 & U & U & M & M & M & M & H & M & M & H & unknown \\
ESM2 & Ma & T & M & M & M & N & H & M & M & M & absent \\
BayShore3 & BS & CS & M & M & M & M & H & M & M & M & sulfide \& sulfate \\
BayShore4 & BS & CS & M & M & M & M & H & M & M & M & sulfide \& sulfate \\
Sayville5 & S & T & N & M & N & N & H & N & N & M & minute sulfide \\
Sayville7 & S & T & M & M & L & M & H & N & N & M & unknown \\
Sayville8 & S & T & N & M & N & N & H & N & M & M & unknown \\
WestIslipA & WI & T & L & M & L & N & M & M & L & H & sulfide \& sulfate \\
WestIslipB & WI & CS & M & M & M & N & H & M & M & M & sulfide \& sulfate \\
\hline
\end{tabular}

Locations listed as BS = Bay Shore, NY, USA, S = Sayville, NY, USA, WI = West Islip, NY, USA; Ma = Manorville, NY, USA, Mo = Morocco, Africa, U = unknown, $\mathrm{K}=$ Kansas, USA, WA = West Australia; Meteorite Class listed as U $=$ Undocumented meteorite, $\mathrm{CS}=$ cosmic spherule, $\mathrm{T}=$ terrestrial, $\mathrm{CC}=$ Carbonaceous Chondrite; $\mathrm{A}=$ Aubrite meteorite; Fluorescence signals are ranked as $\mathrm{H}=$ high (greater than $10^{7}$ counts), $\mathrm{M}=$ medium $\left(10^{5}-10^{7}\right.$ counts), $\mathrm{L}=$ low (less than $10^{5}$ counts), $\mathrm{N}=$ none observed (no counts).

\subsection{Data Collection}

Collected samples and reference meteorites were taken to the National Synchrotron Light Source II (NSLS-II) at Brookhaven National Laboratory (BNL). Meteorites were used as an example of extraterrestrial reference materials and were provided by D. Meisel. Samples were analyzed by X-rays for chemical and mineral determination at the submicron resolution X-ray spectroscopy (SRX) beamline 5-ID, at the tender energy X-ray absorption spectroscopy (TES) beamline 8-BM, and at the X-ray fluorescence microprobe (XFM) beamline 4-BM.

\subsubsection{X-ray Fluorescence to Determine Elemental Composition}

Synchrotron X-ray fluorescence imaging method uses a microfocused X-ray beam to excite characteristic fluorescence from various elements in the sample. Fluorescence is collected by an energy-dispersive detector to separate the different elements' signals. Raster-scanning the sample across the X-ray beam generates a map of elemental distributions and relative abundances. SRX, an undulator beamline with incident beam energy selected by a double crystal $\mathrm{Si}$ (111) monochromator, has XRF imaging capabilities at energies ranging from $4.65-25 \mathrm{keV}$. The beam is focused using a set of Kirkpatrick-Baez mirrors to a spot size of $\sim 2 \mu \mathrm{m}$ [25]. For each sample and several reference meteorites, X-ray fluorescence images were obtained, and spectra were summed across an area of approximately $40 \mu \mathrm{m}^{2}$ at an incident beam energy of $12 \mathrm{keV}$ to capture the potential presence of transition metals, 
including iron, nickel, copper, and zinc. The spectra were analyzed using a python-based fluorescence analysis program (PyXRF) [26] to fit the X-ray fluorescence data for visualization.

\subsubsection{XRF and XANES of Light Elements}

X-ray absorption spectroscopy provides further element-specific information about oxidation state and chemical species. By scanning the incident beam energy across an element's absorption edge, while measuring its fluorescence, one obtains a spectrum whose detailed structure can be used to fingerprint a chemical species and identify its oxidation state. This is often used in combination with XRF imaging, such that areas of high concentration are located by XRF and their chemical species then probed by XANES. The TES beamline operates at lower energy (2.0-5.5 keV, the "tender" X-ray energy range) than SRX, with a beam size adjustable from $5 \times 20$ to $2 \times 2 \mu \mathrm{m}$. In order to measure MMs and meteorite references NWA 3118 (CC) and Hamada (undocumented meteorite), a beam size of $4 \times 12 \mu \mathrm{m}$ was utilized. This allowed lighter elements, specifically sulfur, to be detected. Details of the TES beamline are described in Northrup [27]. An overall XRF map of the sample was run first to see hotspots of signal intensity for sulfur in the MM candidate samples and the reference meteorites. Quick maps were conducted at a $25-\mu \mathrm{m}$ resolution and at $0.1 \mathrm{~s}$ per pixel. Beamline-specific software (written in Interactive Data Language, IDL) was used to create maps with visual representations of elements present in each sample as well as the relative elemental intensities. After selecting candidate spots on each sample through XRF imaging at an incident energy of $2700 \mathrm{eV}$, sulfur k-edge XANES analysis was conducted to determine speciation as sulfate or sulfide. The incident beam energy was scanned from 2442 to $2580 \mathrm{eV} ; 10$ scans were averaged for each point. A gypsum sample was analyzed with the same parameters to serve as a sulfate standard. Data were analyzed using Athena software [28].

\subsubsection{X-ray Microdiffraction}

Mineral components were identified at the XFM beamline. The beam was focused to $9 \mu \mathrm{m}$ in size at an energy of $18 \mathrm{keV}$ [29]. A LaB6 standard was used for calibration. The diffractogram patterns were processed and analyzed using Dioptas software [30] and Match! [31] software using d-spacing to match minerals in the American Mineralogist Crystal Structure database [32]. The XFM beamline was utilized because microbeam diffraction was necessary for small samples. Initial attempts to use a bulk powder diffraction beamline found that the beam was larger than the samples themselves and, as a result of the mounting procedure, the petrolatum coating the samples overwhelmed the relatively weak sample signal.

\section{Results}

\subsection{X-ray Fluorescence of Transition Metals}

All data presented for X-ray fluorescence of transition metals include a scan area of $40 \mu \mathrm{m}^{2}$ analyzed in each particle. Entire particles were not able to be analyzed for the sake of time and resources. We report raw counts, which are a qualitative measure of element content. Iron was observed in all of the particles analyzed in this research as properties of magnetism were used in the selection process (Table 1). All particles recorded high fluorescence signals for iron except for WestIslipA, which recorded a medium fluorescence. Fluorescence signals were ranked so that high was greater than $10^{7}$ counts, medium was a range of $10^{5}-10^{7}$ counts, low fluorescence was less than $10^{5}$ counts, and none observed means the element was not detected. The detector recorded medium fluorescence signals for nickel in most of the particles except for the Inkerston (undocumented meteorite) and all of the Sayville particles. Additional metal fluorescence signals were recorded as medium, low, or none for elements including $\mathrm{Ca}, \mathrm{Ti}, \mathrm{Cr}, \mathrm{Mn}, \mathrm{Cu}$, and $\mathrm{Zn}$, as noted in Table 1.

Mt. Egerton, a meteorite; BayShore3, a micrometeorite; and Sayville5, a terrestrial particle are presented as representatives of their respective particle types. Figure 4 shows the XRF spectra for identification of elements in each representative sample. The XRF spectra are plotted in a log scale to show the relative intensity for the elements analyzed. For example, in the Mt. Egerton meteorite, 
there was relatively high fluorescence for iron as compared to $\mathrm{Ni}, \mathrm{Cu}$, and $\mathrm{Zn}$. In the micrometeorite BayShore3, there was relatively medium fluorescence for $\mathrm{Cu}$ and $\mathrm{Zn}$ but still less than iron, which was recorded as high fluorescence. In the terrestrial particle, Sayville5, iron fluorescence was recorded as high but in terms of thousands of counts, iron was close to $10^{9}$ counts while iron in the meteorite and micrometeorite samples were on the order of $10^{8}$ counts.

Selected elemental maps are shown in Figure 5. Here, we can see how the elements of interest are distributed and their relative intensity within the $40-\mu \mathrm{m}^{2}$ scan area for each particle. Hot spots for each element are on the red end of the spectrum and intensity counts are indicated with a number scale on the right of the image. For example, on the area scan of the meteorite Mt. Egerton (Figure 4a), there are over two million counts of iron in the upper left corner. In the same location, nickel can be seen at counts of over 4500 and a small spot of titanium is seen at a count of over 32,000. Copper does not appear red; the green color indicates a lesser count of copper of around 1000 counts. Fluorescence for these elements cannot be seen in the blue areas of the scan. The scan for the micrometeorite representative BayShore3 shows high-intensity spots of iron that are more scattered along with less intense counts of $\mathrm{Ni}, \mathrm{Cu}$, and Ti. In contrast, the Sayville5 terrestrial particle area scan indicates high fluorescence of iron at counts reaching 33,000,000 and titanium reaching 5400. Both elements are present throughout the entire area scan and can be seen in the same configuration.

Tri-Color elemental maps of red, green, and blue each indicate a specific element (Figure 6). The meteorite representative shows iron as red, nickel as green, and titanium as blue. All three elements can be seen in the same location in the sample in the upper left corner, which is why the larger picture is represented as a mixture of the three colors (Figure 6a). Conversely, the micrometeorite shows spots of each element scattered throughout the sample with no colocalization (Figure 6b). Iron and titanium were mapped in the terrestrial particle as nickel was not present. In the Sayville5 terrestrial sample, we see red iron and green titanium colocalized throughout the sample, which is why they appear yellow, while there are small spots of blue zinc, which is not colocalized with iron and titanium (Figure 6c). 
Meteorite (Mt. Egerton)

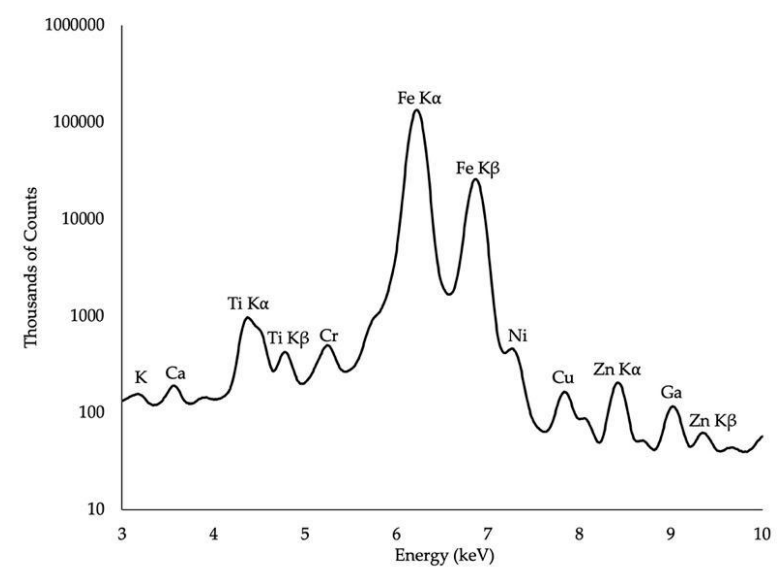

(a)

Micrometeorite (BayShore3)

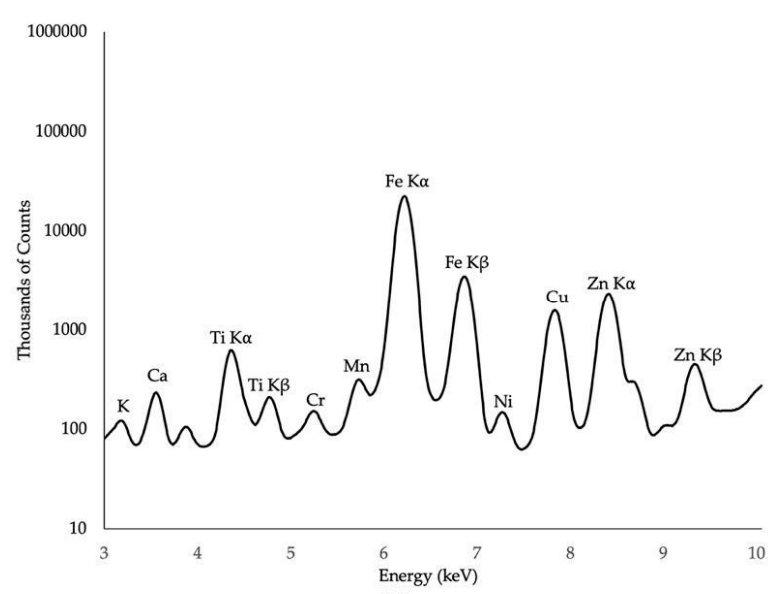

(b)

Terrestrial Particle (Sayville5)

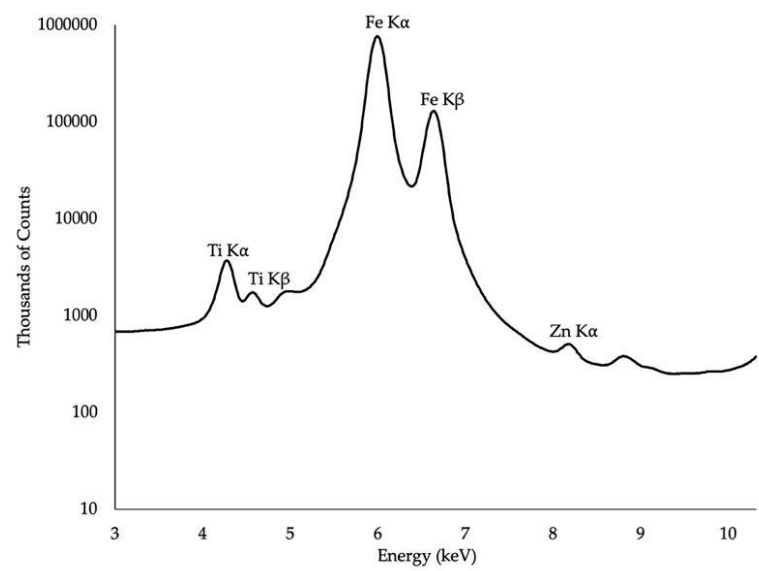

(c)

Figure 4. X-ray fluorescence (XRF) spectra of elements present in (a) Mt. Egerton (Aubrite) meteorite; (b) BayShore3 collected particle identified as a micrometeorite and classified as a cosmic spherule (CS), and (c) Sayville5, collected particle identified as terrestrial origin. Fluorescence peaks for those elements identified are labeled. Note the similarity between the elements identified and their relative counts for (a) meteorite and (b) micrometeorite while (c) the terrestrial particle has an absence of $\mathrm{Ni}$ and higher counts of $\mathrm{Ti}$ and $\mathrm{Fe}$ as compared to the extraterrestrial particles. 


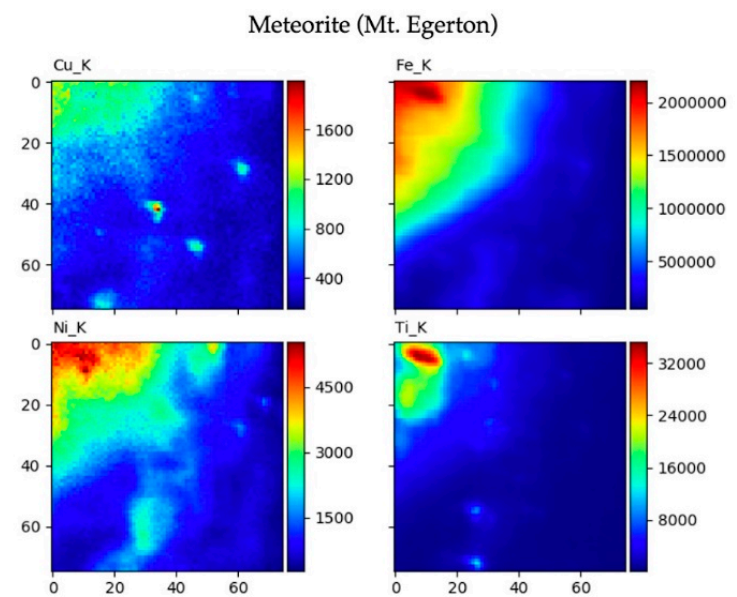

(a)

Micrometeorite (BayShore3)
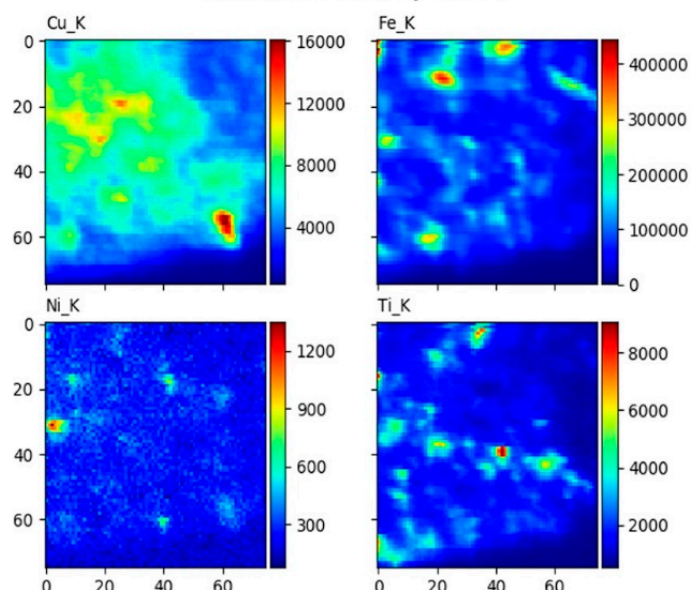

zn_k

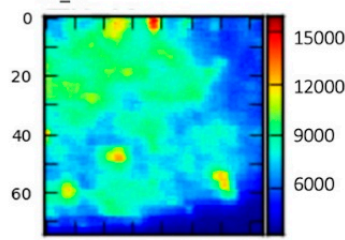

(b)

Terrestrial Particle (Sayville5)
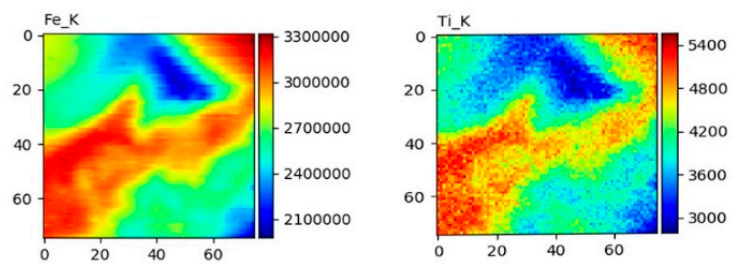

(c)

Figure 5. Spectral maps are a 2-D scan of the intact particle. The scan area shows key elements, including copper, iron, nickel, titanium, and zinc, in representative samples. These maps use color to show the location of the elements within the $40 \times 40 \mu \mathrm{m}$ scan area of each sample as well as relative numbers of counts for each element. The red indicates spots of high counts for the element while a blue color is low counts. Mount Egerton (a), a documented meteorite, contains a similar chemical structure to the BayShore3 micrometeorite (b) while Sayville5 (c) is terrestrial in origin due to a lack of key elements being present in extraterrestrial samples. 
Meteorite (Mt. Egerton)

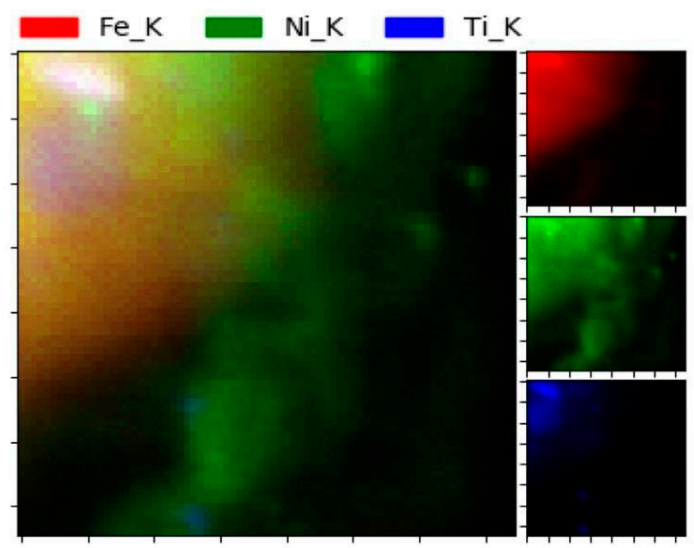

(a)

Micrometeorite (BayShore3)

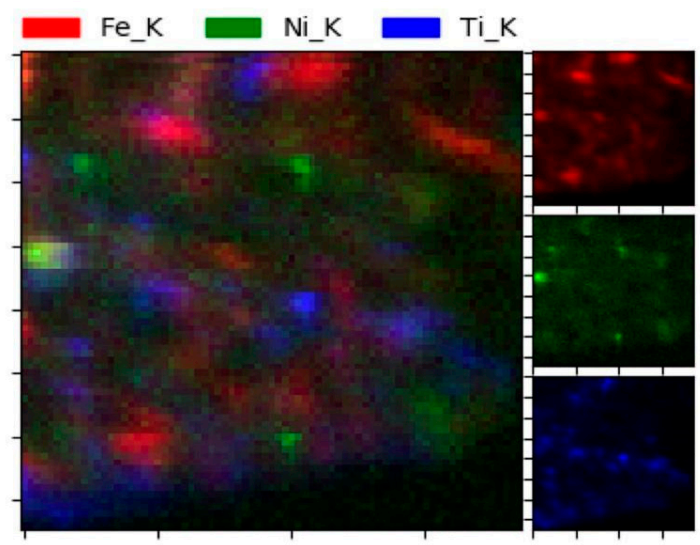

(b)

Terrestrial Particle (Sayville5)

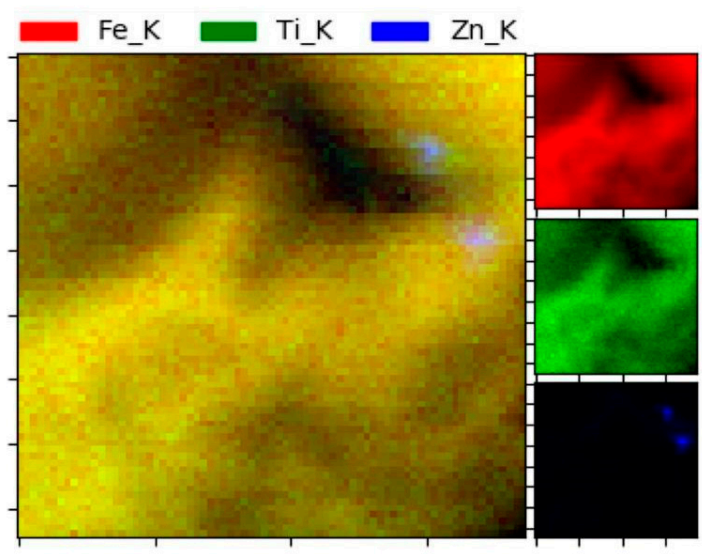

(c)

Figure 6. Tricolor maps are a 2-D scan of the intact particle. The scan area shown is $40 \times 40 \mu \mathrm{m}$. The colors of red, green, and blue are assigned to elements, respectively, and show areas of elemental colocalization. Mt. Egerton (a) shows colocalization of iron (red), nickel (green) and titanium (blue). Micrometeorite BayShore3 (b) shows that the elements are scattered. Conversely, Sayville5 (c) contains no nickel while iron (red) and titanium (green) are colocalized, appearing yellow throughout the entire area analyzed, while zinc (blue) appears in small light spots on the sample. Note the fine-grained texture of BayShore3 as compared to Mt. Egerton. 


\subsection{TES Analysis for Light Elements}

The major interest in performing spectroscopic measurements at tender X-ray energies, $2.0-5.5 \mathrm{keV}$, was to identify sulfur species in the samples (Table 1). XRF images of BayShore3 and WestIslipB were measured at an incident beam energy of $2700 \mathrm{eV}$ to show the sulfur fluorescence within the sample (Figures 7 and 8). XRF maps were conducted to determine the sulfur distribution. Based on this distribution, areas of high counts were selected to conduct XANES analysis in one spot of sulfur. This data indicated the presence of sulfate at $2482.5 \mathrm{eV}$, as compared to a gypsum standard, while peaks occurring at lower energy are broadly categorized as sulfides (Figure 9). Samples BayShore3 and BayShore4 contained strong concentrations of both sulfide and sulfate. Samples WestIslipA and WestIslipB showed the presence of sulfide only. Sayville5 also contained sulfide. The meteorite references Hamada and NWA 3118 showed sulfide abundance but in a slightly different form than that observed in any of the other particles containing sulfide (Figure 9).

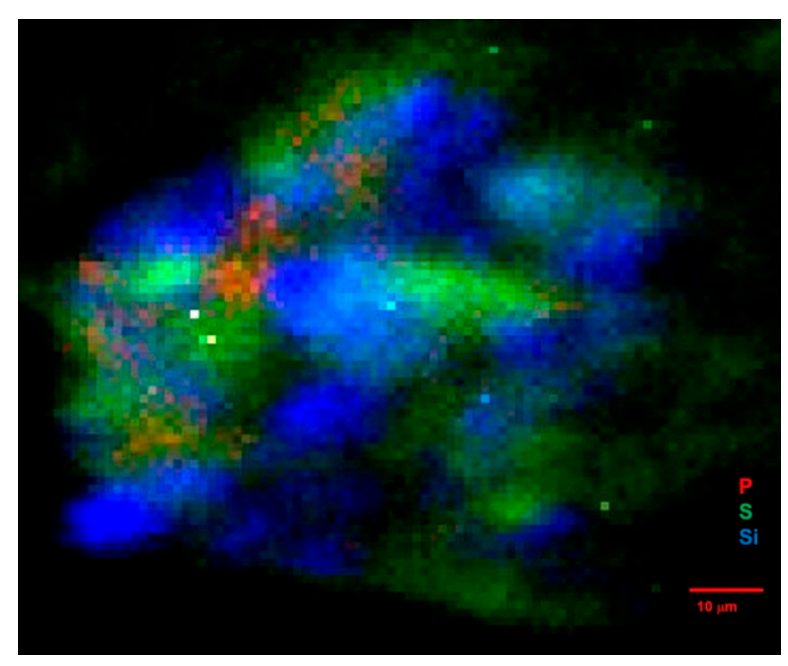

Figure 7. Complete XRF image of cosmic spherule BayShore3; this tricolor map shows $\mathrm{P}$ (red), $\mathrm{S}$ (green), and $\mathrm{Si}$ (blue). Note the elements are distributed heterogeneously throughout the particle.

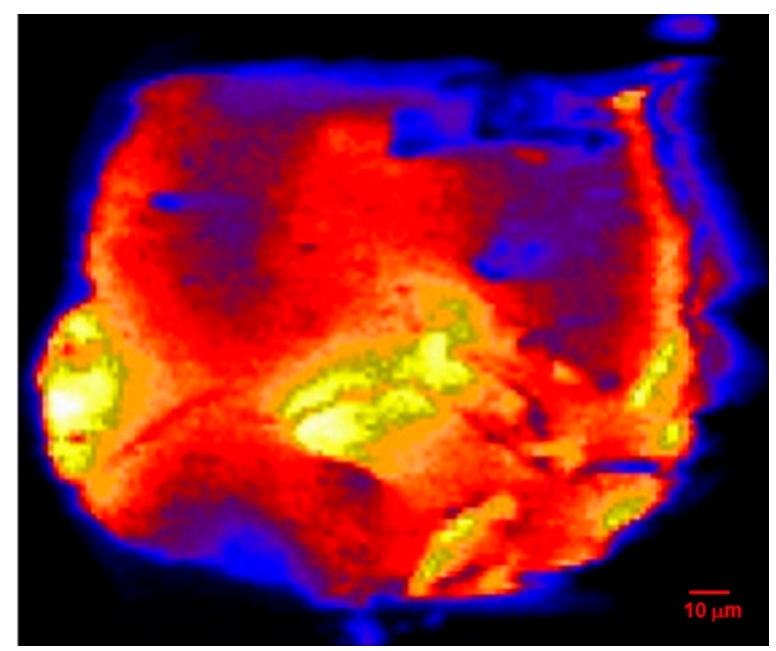

Figure 8. Complete XRF image of cosmic spherule WestIslipB depicts the sulfur distribution throughout the micrometeorite surface; yellow depicts the highest relative amounts of $S$ while blue depicts the lowest relative amounts of S. Sulfur distribution is similar in the maps of both micrometeorites in Figures 7 and 8. 


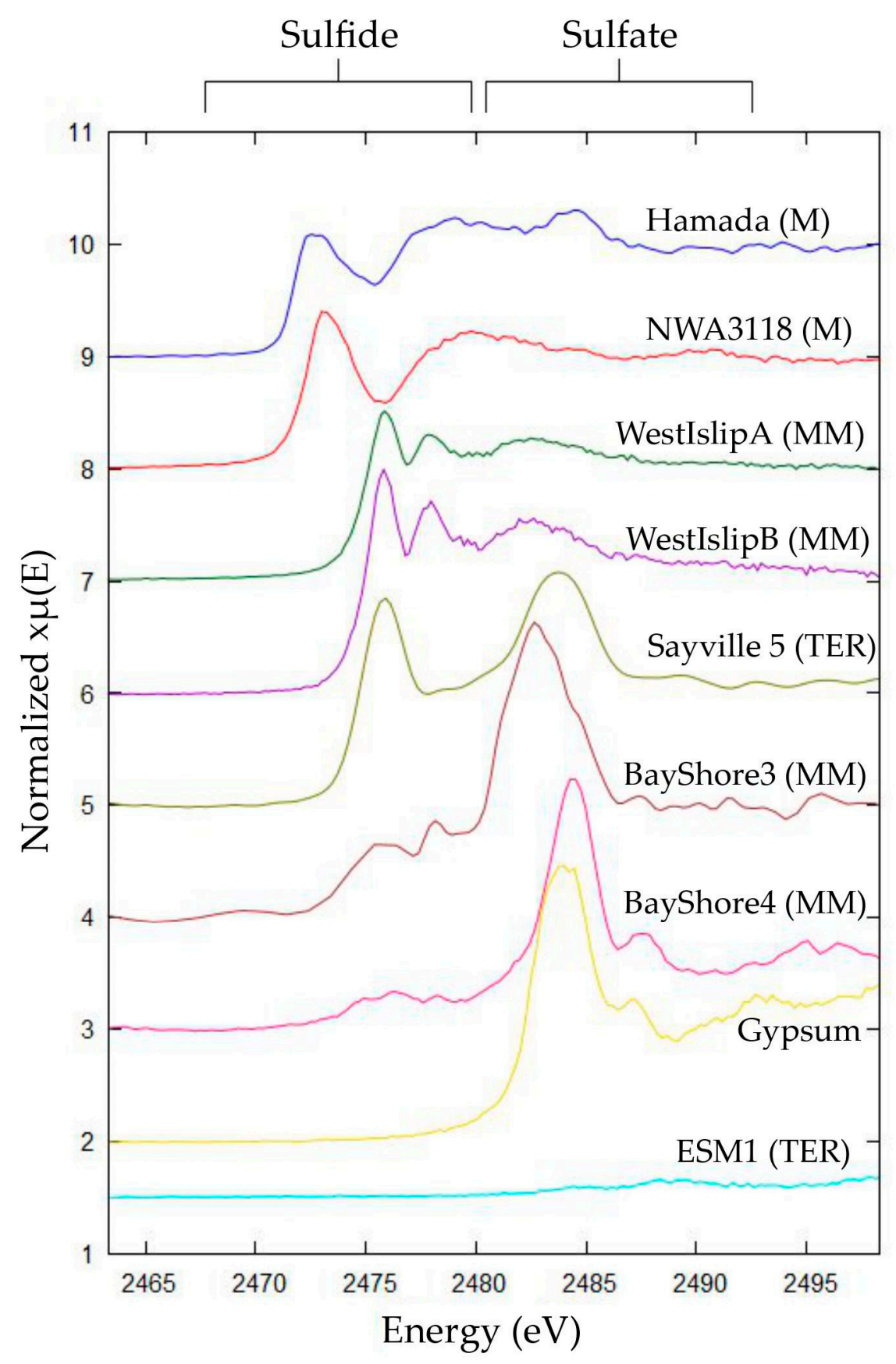

Figure 9. Sulfur X-ray absorption near-edge structure (XANES) spectra using tender energy X-ray absorption spectroscopy (TES) indicating sulfur speciation in meteorites (Ms), micrometeorites (MMs), and terrestrial particles (TERs). Gypsum was used as a standard for sulfate. The left-shifted peaks are representative of possibly extraterrestrial sulfide species of sulfur.

\subsection{Analysis by X-ray Microdiffraction}

The powder diffraction data collected at the XFM microprobe beamline allowed the identification of forsterite and pentlandite in BayShore3. Both minerals are frequently observed in micrometeorites (Figure 10) [11,13,14]. 


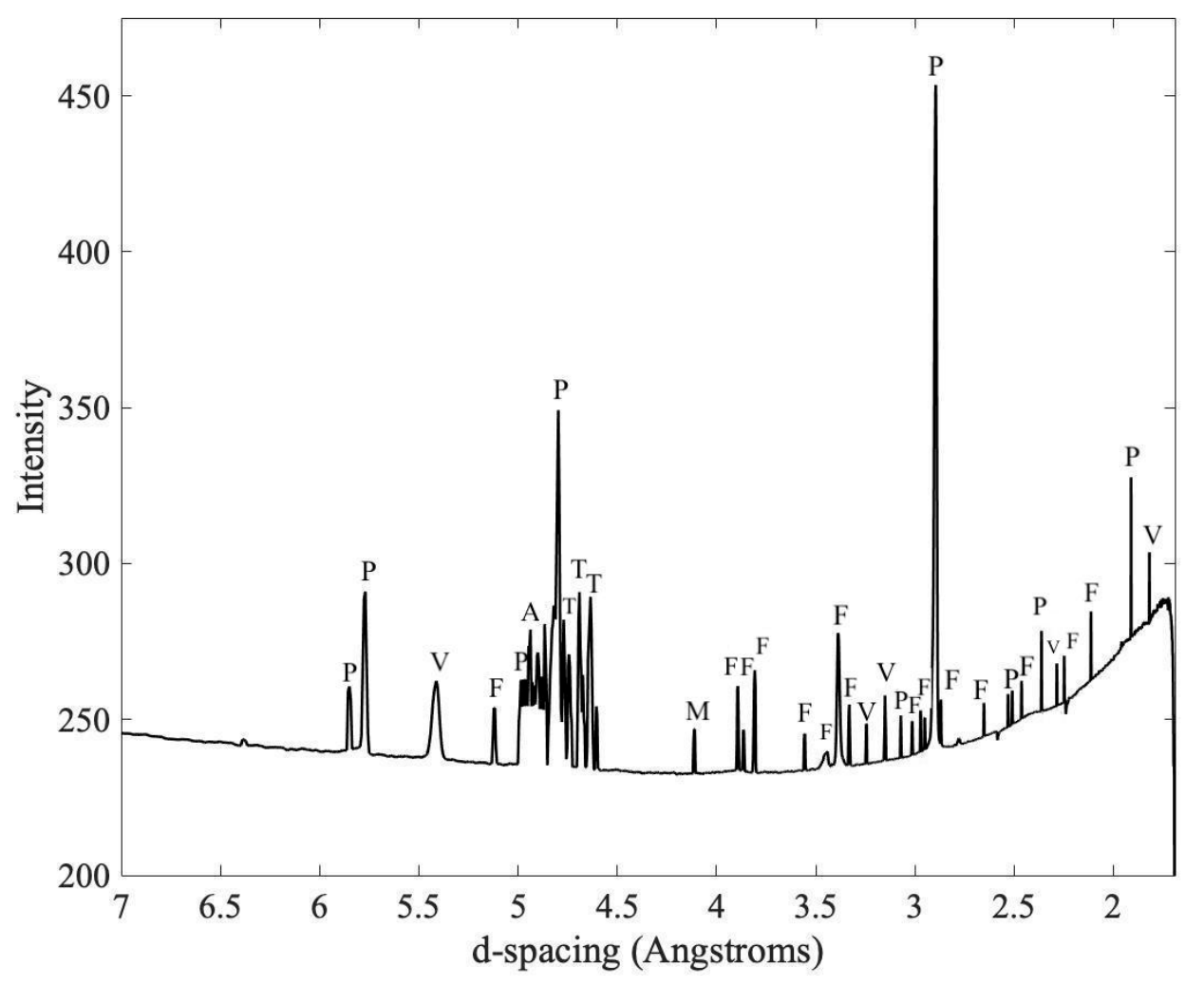

Figure 10. Microdiffraction powder pattern CS BayShore3: Pentlandite (P), forsterite (F), violarite (V), troilite $(\mathrm{T})$, alunite $(\mathrm{A})$, and melanterite $(\mathrm{M})$. The predominant presence of pentlandite and forsterite peaks are indicative of extraterrestrial origin [12-15].

\section{Discussion}

Cosmic spherules are expected to have Ni-bearing iron metal, magnesium-rich olivine (forsterite), near chondritic chemical composition, heterogeneity due to relict grains, and Fe-bearing sulfides $[1,7,11]$. Based on physical sample selection criteria, XRF, XANES, and XRD results, the tested particles were categorized by their chemical properties as cosmic spherules (Table 2) or of terrestrial origin. The three cosmic spherules characterized in this research are round, magnetic, and approximately between 50 and $500 \mu \mathrm{m}$ in diameter as described in Genge et al. (2008) and Noguchi et al. (2000) [1,24]. I-type (iron rich and magnetic) CS can contain Fe-Ni beads and are proven to be easily collected from modern sediments [33,34]. The absence of both nickel and copper was used as an indicator of terrestrial origin [1], as the presence of chondritic Fe-Ni and sulfides strongly supports extraterrestrial origin [15]. Particles determined to be likely extraterrestrial origin were found to contain forsterite; forsterite and pentlandite are recognized as extraterrestrial relict minerals [11-14,35].

XRF qualitative data for iron and nickel in three of the collected particles, CS BayShore3, CS BayShore4, and CS WestIslipB, are consistent with previously reported data for cosmic spherules [1,7]. The $40-\mu \mathrm{m}^{2}$ imaging area (constrained by SRX instrumentation and limited beam availability) and the heterogeneity of MM samples prevent accurate identification of minor elemental ratios based on XRF data. The heterogeneity of metallic elements is presented in Figures 5 and 6. For example, CS BayShore3 (Figure 5b) shows a wide distribution of copper and zinc within the small area scanned, whereas iron is present in only part of this area, leading to an artificially high relative amount of copper and zinc, more than the smaller amounts typically found in meteorites (e.g., Mt. Egerton in Figure 4 and others in Table 1). Cosmic spherules show different mineral concentrations in grains when they are analyzed by scanning electron microscope (SEM) imaging and by energy dispersive $X$-ray spectroscopy (EDS) $[7,11,15]$. Figure 6a shows the colocalization of iron and nickel within the meteorite Mt. Egerton, while Figure $6 \mathrm{~b}$ shows the separation of the same elements within BayShore3 supporting 
the CS heterogeneity. Taken together, XRF imaging and SEM imaging with EDS are complementary to each other.

Table 2. Total samples collected and characterized as extraterrestrial in origin after synchrotron analysis.

\begin{tabular}{|c|c|c|c|c|c|c|}
\hline $\begin{array}{c}\text { Total } \\
\text { Samples } \\
\text { Collected }\end{array}$ & $\begin{array}{l}\text { Sample } \\
\text { Selection } \\
\text { Criteria }\end{array}$ & $\begin{array}{c}\text { Total } \\
\text { Samples } \\
\text { Tested }\end{array}$ & $\begin{array}{c}\text { SRX } \\
\text { Characterization } \\
\text { Criteria }\end{array}$ & $\begin{array}{c}\text { TES } \\
\text { Characterization } \\
\text { Criteria }\end{array}$ & $\begin{array}{c}\text { XFM } \\
\text { Characterization } \\
\text { Criteria }\end{array}$ & $\begin{array}{l}\text { Total Samples } \\
\text { Characterized as } \\
\text { Cosmic Spherules }\end{array}$ \\
\hline $100+$ & $\begin{array}{c}\text { Magnetic } \\
\text { spherical shape } \\
\text { particle diameter } \\
\sim 100 \mu \mathrm{m}\end{array}$ & 8 & $\begin{array}{l}\text { Fe intensity high } \mathrm{Ni} \\
\text { intensity consistent } \\
\text { with meteorites } \\
\text { Absence of } \\
\text { disqualifying } \\
\text { terrestrial components }\end{array}$ & $\begin{array}{l}\text { Sulfide and sulfate } \\
\text { presence } \\
\text { consistent with } \\
\text { meteorite samples }\end{array}$ & $\begin{array}{l}\text { Presence of } \\
\text { minerals known to } \\
\text { be extraterrestrial } \\
\text { including } \\
\text { forsterite and } \\
\text { pentlandite }\end{array}$ & 3 \\
\hline
\end{tabular}

Simple diagnostic analysis was applied to disqualify samples with terrestrial features. Zinc was detected within all samples, including in small amounts within the mounting material. A much larger quantity of zinc was detected in WestIslipA as compared to a relatively small iron signal; this overwhelming quantity, as well as a large quantity of titanium, indicated that the sample was not a micrometeorite. High titanium content in relation to iron is not consistent with what is found in MMs [1]. Two samples from Sayville were determined to be terrestrial particles as they contained a large amount of titanium and no nickel, which suggests terrestrial origin (Figure 5).

Sulfur k-edge XANES were measured in candidate particles to further differentiate between terrestrial and extraterrestrial particles. Hard X-ray XRF analysis at the SRX beamline, for example, of ESM2, showed a metallic composition consistent with MM. XRF maps at TES indicated high sulfur content; however, XANES showed that ESM2 had very little sulfur, indicating a high concentration of lead due to M-alpha fluorescence being misidentified as sulfur K-alpha fluorescence, since these signals overlap. The overwhelming presence of lead as opposed to sulfur is unlikely for an extraterrestrial source [36]. The distinction between the use of hard and tender energy $\mathrm{X}$-rays is highlighted in this example, as without the tender energy X-rays used for XANES at TES, this sample may have been misidentified as MM, showing the value in the use of multiple synchrotron analysis.

The heterogeneity of sulfur across the entire CS WestIslipB is shown in Figure 8. The presence of sulfide is consistent with extraterrestrial origin, as the main constituent of sulfur in many types of meteorite is troilite, an iron sulfide [37], and pentlandite, an iron nickel sulfide [14]. Samples still showed the presence of some sulfate, but this can be attributed to the weathering effects of extended time submerged in water in the collection tubs and does not exclude potential extraterrestrial origin [38]. The forms of sulfide existing within the meteorite standards suggest a difference in sulfide speciation between the meteorites and the micrometeorites. However, the presence of sulfide in any form in these samples is suggestive of extraterrestrial nature (Figure 9) $[15,20]$. The sulfide species revealed in analyzed particles have yet to be exactly matched with species in sulfur databases; however, we interpret this as follows. The difference in sulfide phases between MMs and our compared meteorite standards is a result of the small size of MMs, which can lead to melting and mixing with Fe-Ni metal to result in minerals like pentlandite and troilite (Figure 10) [11-14,20,35]. The intermediate and oxidized phases present in our MM samples can be ascribed to weathering processes after atmospheric entry [38]. Though Sayville5 was characterized as terrestrial based on SRX analysis, XANES revealed minute sulfide within the sample, which requires further investigation. XRF mapping at TES shows heterogeneity of sulfur, silicon, and phosphorus content across CS BayShore3. XANES further revealed the CS BayShore3 sulfur content was in the form of sulfide (Figure 9), which is consistent with the CS classification [20] and sulfate, which may have been a result of weathering [38]. Powder microdiffraction used for mineral identification revealed forsterite and pentlandite in CS BayShore3, which further supports its characterization as CS (Figure 10) [11-14,35]. Elemental distributions, speciation, and relative abundance data from SRX and TES in combination with XFM microdiffraction data show a clearer picture of extraterrestrial versus terrestrial nature of collected samples. 


\section{Implications for Research in High School Science Education}

Teachers and students in school districts across Long Island researched MM in collaboration with Brookhaven National Laboratory (BNL) and State University of New York (SUNY) scientists as part of BNL's SPARK (Student Partnerships Advancing Research and Knowledge) program. The program allowed teachers and students from across Long Island to develop and conduct this MM study among other spectroscopy-centered student and teacher partnerships.

Students involved have been collaborating within the program for multiple years and work with incoming students to ensure continuity and future success. The scientific community is able to see the impact of this experiment as researchers bring this research to various science fairs and symposia, aim to publish their research, and share their findings with their schools and communities. Collaboration between high schools further broadens the reach of this project, as more students have the opportunity to become involved in multiple stages of research. This project has served as an example of a successful collaboration between the NSLS-II and the Office of Educational Programs at BNL, and local high schools in the education and development of the next generation of STEM professionals. The involvement of high school students in research activities beyond the scope of their regular curriculum has a significant and lasting positive impact on their lives and careers.

\section{Conclusions}

Characterizing the origin of particles collected on suburban rooftops as extraterrestrial requires more than one method of chemical and structural analysis. Synchrotron X-ray fluorescence imaging at different energies enables the observation and distribution of key elements in CS, including iron, nickel, and sulfur. X-ray absorption near-edge structure reveals the speciation of chemical elements, for example, sulfur was determined to be in the form of sulfide in extraterrestrial particles. Synchrotron $X$-ray microdiffraction was used to identify minerals; pentlandite and forsterite were identified in the CS. All together, these X-ray methods allow for the determination of elemental composition, speciation, and mineralogy of the CS. Synchrotron analysis gives us new insight into ways to analyze extraterrestrial materials. This also has advantages not available to SEM, such as non-destructive sample preparation, chemical speciation through XANES, and compositional sampling below the surface of particles. Synchrotron technology can complement traditional SEM as in Dionnet et al. (2020) [22] and may provide the best outcomes for extraterrestrial identification.

Conducting authentic research from project inception through to the communication of results is impactful learning for high school students. They collaborated with scientists and teachers to design the experimental protocols and then used advanced tools at a scientific institution to collect and analyze data. Exposing students to cutting-edge research tools and real-world science motivates them to pursue careers in science, technology, engineering, and mathematics.

Author Contributions: Conceptualization, M.E. and K.S.; Data curation, R.B., J.K. and D.M.; Formal analysis, M.E., K.S., E.G., M.K., P.N., V.S., J.T. and A.P.; Investigation, M.E., K.S., E.G., M.K., P.N., V.S., J.T. and A.P.; Methodology, M.E., K.S., E.G. and M.K.; Resources, V.S., J.T. and A.P.; Supervision, V.H., A.S. and J.T.; Validation, P.N., V.S., J.T. and A.P.; Writing —original draft, E.G., V.H., P.N., V.S. and A.P.; Writing—review \& editing, V.H. All authors have read and agreed to the published version of the manuscript.

Funding: National Synchrotron Light Source II, a U.S. Department of Energy (DOE) Office of Science User Facility operated for the DOE Office of Science by Brookhaven National Laboratory under Contract No. DE-SC0012704. Additional support was provided by the Office of Educational Programs at Brookhaven National Laboratory, West Islip High School, Bay Shore High School, William Floyd High School, Sayville High School, Eastport South Manor High School, and State University of New York at Stony Brook Science and Technology Entry Program.

Acknowledgments: We would like to acknowledge the support of the NSLS-II staff, specifically of beamlines SRX 5-ID, TES 8-BM, XFM 4-BM, and XPD 28-ID-2 especially E. Dooryhee, J. Bai, and D. Olds for assistance with XPD data collection and analysis. We would also like to acknowledge the NSLS-II User Office, L. Miller, G. Cisco, G. McKenzie and the Office of Educational Programs, K. White and S. Bronson for support of the SPARK program. A special thank you to N. Marinkovic for assistance with beam time at the TES beamline. We would like to acknowledge the New York State Master Teacher Program for supporting teacher participation in collaboration with the SPARK program and all teachers in the High School Spectroscopy Collaboration; L. Hemmick for helping 
with sample collection at Longwood High School; and K. LaSpisa, M. Borrayo and A. Pecorale for assistance with data analysis.

Conflicts of Interest: The authors of this publication declare no conflict of interest.

\section{References}

1. Genge, M.J.; Engrand, C.; Gounelle, M.; Taylor, S. The classification of micrometeorites. Meteorit. Planet. Sci. 2008, 43, 497-515. [CrossRef]

2. Suavet, C.; Gattacceca, J.; Rochette, P.; Perchiazzi, N.; Folco, L.; Duprat, J.; Harvey, R.P. Magnetic properties of micrometeorites. J. Geophys. Res. Solid Earth 2009, 114, B4. [CrossRef]

3. Suttle, M.D.; Folco, L. The extraterrestrial dust flux: Size distribution and mass contribution estimates inferred from the Transantarctic Mountains (TAM) micrometeorite collection. J. Geophys. Res. Planets 2020. [CrossRef]

4. Maurette, M.; Olinger, C.; Michel-Levy, M.C.; Kurat, G.; Pourchet, M.; Brandstatter, F.; Bourot-Denise, M. A collection of diverse micrometeorites recovered from 100 tonnes of Antarctic blue ice. Nature 1991, 351, 44-47. [CrossRef]

5. Duprat, J.; Engrand, C.; Maurette, M.; Kurat, G.; Gounelle, M.; Hammer, C. Micrometeorites from central Antarctic snow: The CONCORDIA collection. Adv. Space Res. 2007, 39, 605-611. [CrossRef]

6. Dobrica, E.; Engrand, C.; Duprat, J.; Gounelle, M. A statistical overview of concordia Antarctic micrometeorites. In Proceedings of the 73rd Annual Meeting of the Meteoritical-Society, New York, NY, USA, 26-30 July 2010.

7. Taylor, S.; Matrajt, G.; Guan, Y. Fine-grained precursors dominate the micrometeorite flux. Meteorit. Planet. Sci. 2012, 47, 550-564. [CrossRef]

8. Taylor, S.; Lever, J.H.; Harvey, R.P. Accretion rate of cosmic spherules measured at the South Pole. Nature 1998, 392, 899-903. [CrossRef]

9. Taylor, S.; Lever, J.H.; Harvey, R.P. Numbers, types, and compositions of an unbiased collection of cosmic spherules. Meteorit. Planet. Sci. 2000, 35, 651-666. [CrossRef]

10. Brownlee, D.E. Cosmic dust: Building blocks of planets falling from the sky. Elements 2016, 12, 165-170. [CrossRef]

11. Imae, N.; Taylor, S.; Iwata, N. Micrometeorite precursors: Clues from the mineralogy and petrology of their relict minerals. Geochim. Cosmochim. Acta 2013, 100, 116-157. [CrossRef]

12. Steele, I.M. Olivine in Antarctic micrometeorites: Comparison with other extraterrestrial olivine. Geochim. Cosmochim. Acta 1992, 56, 2923-2929. [CrossRef]

13. Beckerling, W.; Bischoff, A. Occurrence and composition of relict minerals in micrometeorites from Greenland and Antarctica-Implications for their origins. Planet. Space Sci. 1993, 43, 435-449. [CrossRef]

14. Singerling, S.A.; Brearley, A.J. Primary iron sulfides in CM and CR carbonaceous chondrites: Insights into nebular processes. Meteorit. Planet. Sci. 2018, 53, 2078-2106. [CrossRef]

15. Genge, M.J.; Larsen, J.; Van Ginneken, M.; Suttle, M.D. An urban collection of modern-day large micrometeorites: Evidence for variations in the extraterrestrial dust flux through the Quaternary. Geology 2017, 45, 119-122. [CrossRef]

16. Larsen, J. The Stardust Project. In Search of Stardust: Amazing Micrometeorites and Their Terrestrial Imposters; Voyageur Press: Minneapolis, MN, USA, 2017.

17. City Stardust, Micrometeorites in Our Own Backyard. Available online: https://www.bellmuseum.umn.edu/ city-stardust/ (accessed on 26 June 2020).

18. Berlin Collects Cosmic Dust. Available online: https://www.museumfuernaturkunde.berlin/en/science/ berlin-collects-cosmic-dust (accessed on 16 June 2020).

19. Blake, M.; McKee, J.; Statom, R.; Qiu, C.; Menapace, F. Evaluating strategies to collect micrometeorites from rainwater for citizen scientists. J. Astron. Earth Sci. Educ. 2018, 5, 151-160. [CrossRef]

20. Taylor, S.; Jones, K.W.; Herzog, G.H.; Hornig, C.E. Tomography: A window on the role of sulfur in the structure of micrometeorites. Meteorit. Planet. Sci. 2011, 46, 1498-1509. [CrossRef]

21. Van Maldeghem, F.; Goderis, S.; Laforce, B.; Soens, B.; De Pauw, E.; Suuronen, J.P.; Van Ginneken, M.; Folco, L.; Debaille, V.; Vince, L.; et al. Characterisation of unmelted micrometeorites using synchrotron-based X-ray analysis. In EGU General Assembly Conference Abstracts, Proceedings of the EGU General Assembly 2018, Vienna, Austria, 4-13 April 2018; EGU: Vienna, Austria, 2018; Volume 20, p. 15929. 
22. Dionnet, Z.; Suttle, M.D.; Longobardo, A.; Rotundi, A.; Folco, L.; Corte, V.D.; King, A. X-ray computed tomography: Morphological and porosity characterization of giant Antarctic micrometeorites. Meteorit. Planet. Sci. 2020. [CrossRef]

23. Google. Long Island, New York. Available online: https://www.google.com/maps/@40.8466814,-72.9634212, 9z (accessed on 1 December 2018).

24. Noguchi, T.; Imae, N.; Nakamura, T.; Nozaki, W.; Terada, K.; Mori, T.; Fukuoka, T.; Nogami, K.; Ohashi, H.; Nozaki, W.; et al. A consortium study of Antarctic micrometeorites recovered from the Dome Fuji Station. Antarct. Meteor. Res. 2000, 13, 270.

25. Submicron Resolution X-ray Spectroscopy. Available online: https://www.bnl.gov/ps/beamlines/beamline. php?r=5-ID (accessed on 21 May 2020).

26. Li, L.; Yan, H.; Xu, W.; Yu, D.; Heroux, A.; Lee, W.K.; Campbell, S.I.; Chu, Y.S. PyXRF: Python-based X-ray fluorescence analysis package. In X-ray Nanoimaging: Instruments and METHODS III; SPIE: San Diego, CA, USA, 2017; Volume 10389, p. 103890 U.

27. Northrup, P. The TES Beamline (8-BM) at NSLS-II: Tender-energy spatially resolved X-ray absorption spectroscopy and X-ray fluorescence imaging. J. Synchrotron Rad. 2019, 26, 2064-2074. [CrossRef] [PubMed]

28. Ravel, B.; Newville, M. ATHENA and ARTEMIS: Interactive graphical data analysis using IFEFFIT. Phys. Scr. 2005, 2005, T115.

29. X-ray Fluorescence Microprobe (XFM). Available online: https://www.bnl.gov/ps/beamlines/beamline.php? $\mathrm{r}=4-\mathrm{BM}$ (accessed on 21 May 2020).

30. Prescher, C.; Prakapenka, V.B. DIOPTAS: A program for reduction of two-dimensional X-ray diffraction data and data exploration. High Press. Res. 2015, 35, 223-230. [CrossRef]

31. Putz, H.; Brandenburg, K.; GbR, M. Phase Identification from Powder Diffraction CRYSTAL IMPACT; Crystal Impact GbR: Bonn, Germany, 2003.

32. Downs, R.T.; Hall-Wallace, M. The American mineralogist crystal structure database. Am. Mineral. 2003, 88, 247-250.

33. Genge, M.J.; Davies, B.; Suttle, M.D.; van Ginneken, M.; Tomkins, A.G. The mineralogy and petrology of I-type cosmic spherules: Implications for their sources, origins and identification in sedimentary rocks. Geochim. Cosmochim. Acta 2017, 218, 167-200. [CrossRef]

34. Rudraswami, N.G.; Prasad, M.S.; Babu, E.V.; Kumar, T.V. Chemistry and petrology of Fe-Ni beads from different types of cosmic spherules: Implication for precursors. Geochim. Cosmochim. Acta 2014, 145, 139-158. [CrossRef]

35. Olinger, C.T.; Maurette, M.; Walker, R.M.; Hohenberg, C.M. Neon measurements of individual Greenland sediment particles: Proof of an extraterrestrial origin and comparison with EDX and morphological analyses. Earth Planet. Sci. Lett. 1990, 100,77-93. [CrossRef]

36. Prasad, M.S.; Rudraswami, N.G.; De Araujod, A.; Babu, E.V.; Kumar, T.V. Ordinary chondritic micrometeorites from the Indian Ocean. Meteorit. Planet. Sci. 2015, 50, 1013-1031. [CrossRef]

37. Kaplan, I.R.; Hulston, J.R. The isotopic abundance and content of sulfur in meteorites. Geochim. Cosmochim. Acta 1966, 30, 479-496. [CrossRef]

38. Van Ginneken, M.; Genge, M.J.; Folco, L.; Harvey, R.P. The weathering of micrometeorites from the Transantarctic Mountains. Geochim. Cosmochim. Acta 2016, 179, 1-31. [CrossRef]

(C) 2020 by the authors. Licensee MDPI, Basel, Switzerland. This article is an open access article distributed under the terms and conditions of the Creative Commons Attribution (CC BY) license (http://creativecommons.org/licenses/by/4.0/). 